\title{
Design and Implementation of the Infant Incubator Intelligent Control System Based on Internet of Things
}

\author{
Tianshun Huang* and Leiming Sun
}

Henan Polytechnic, Zhengzhou, Henan, 450046, China

\begin{abstract}
The Internet of Things (IoT) is widely used in smart home control application system. But this technology is rarely used in baby care and infant incubator. In this research, an intelligent infant incubator system based on IoT is presented in detail. Firstly, the overall architecture of the system is illustrated based on layer framework of IoT. Secondly, the intelligent local gateway is designed on the basis of Zigbee technology and a type of ARM1136J board. Thirdly, the design of the system software is presented which is marked by loading balancing technology, HTTP protocol and XML. The intelligent system showed a good performance in the environmental monitoring during baby care. In the field of baby care, security issues essentially require privacy of residents and safety of families. Thus, a stable and robust security module should be included in the infant incubator intelligent control system. This will be the focus of our future work.
\end{abstract}

Keywords: ARM, Internet of Things (IoT), infant incubator, the local gateway, system design.

\section{INTRODUCTION}

With the development of the human society and the modern technology, the problem of the care for the new born baby has been focused by more and more people. And the traditional infant incubator can only provide a relative safety environment for the new born babies. With the change in the life style, most of the parents are so busy with the work that they have less or no time to take better care of their babies. Considering the situation, the traditional infant incubator may not provide more reliable care to the babies. In this way, a new kind of infant incubator should be researched which can self-adaptively change the environment based on a series of sensors and real-timely monitor the vital signs for the baby. As the technology of Internet and network is studied by more and more scholars, the Internet of Things has been developed.

The Internet of Things (IoT) is a network system based on the Internet and the information technology emerged with the development of the network technology and hardware. The automatic identification and information sharing are achieved by the use of the IoT.

IoT refers to the object in the structure of a network of a unique identifier and their virtual expression. The IoT was put forward by Kevin Ashton in 1999 [1]. The concept of IoT received fame by Auto-ID center at the Massachusetts institute of technology and related market analyst publications [2]. The radio frequency identification (RFID) is often seen as the precondition of IoT. If all objects are equipped with such as identifier in People's Daily life, they can easily manage and count on computer [3]. Labeling the things

*Address correspondence to this author at the Henan Polytechnic, Zhengzhou, Henan, 450046, China; Tel: 13073726401;

E-mail: 975771759@qq.com could be gotten through the near field communication technology, such as barcodes, QR codes and digital watermarking.

Based on the Research of $\mathrm{ABI}$, more than 30 billion equipments will be wirelessly linked to the IoT by 2020 . Like Internet, the IoT also has the potential to change the world and the life style of people [4]. The research on the IoT is still at the basic stage. Therefore, the standard definitions for The IoT are still null for all the scholars to create and study [5].

With the Internet built with various sensors and devices, IoT is used to link the physical and virtual objects on the basis of exploiting data capture and advanced communication technology [6]. Due to this factor, IoT becomes one of the hot research topics in the twenty-first century. Moreover, IP-based wireless technology brought considerable development into Wireless Sensor Network (WSN) which was widely used in many fields, such as air pollution monitoring, forest fires detection and home automation [7], etc.

The IoT usually has the following characteristics. Firstly, the network is overall perception which obtains information objects by adopting the RFID, sensors, and other twodimensional code in the targets. Secondly, the transmission of the information is reliable which means the real-time information can be passed out through the networks and the Internet. Thirdly, the transmission process is intelligent. The massive data and information are going to be processed, by use of cloud computing, and other intelligent computing techniques [8].

In this research, an infant incubator intelligent control system has been proposed which will help in the care and 


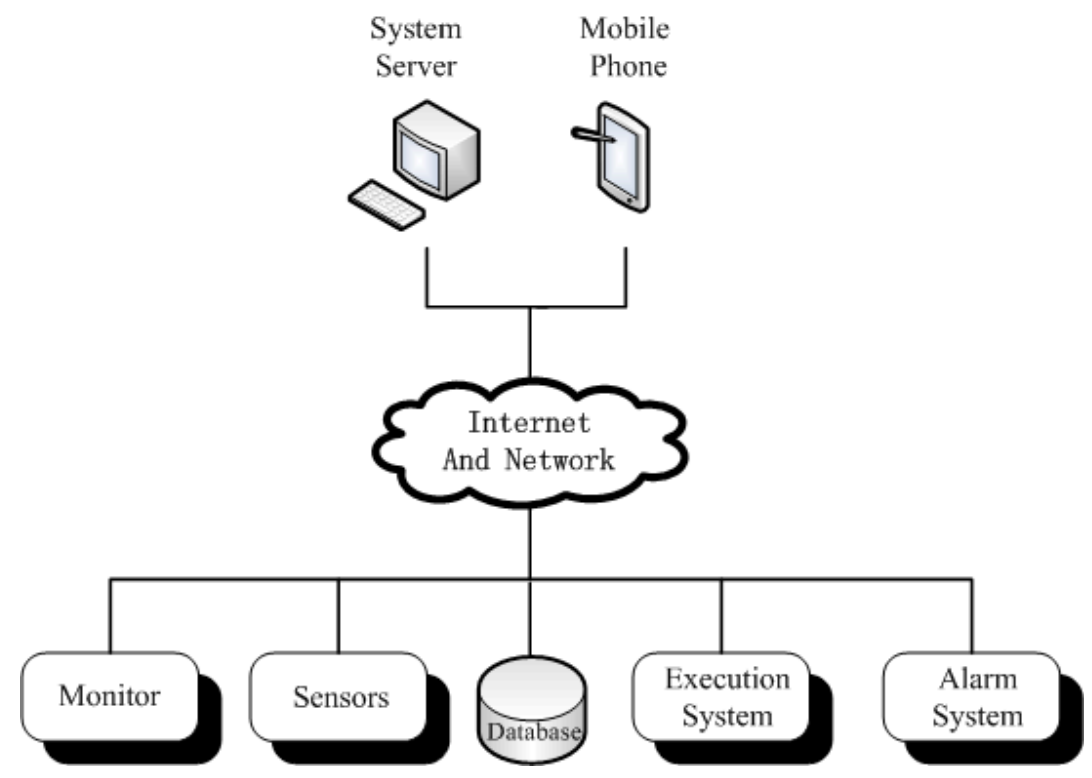

Fig. (1). The overall framework of the system.

monitoring of the new born baby. The hardware and software are designed in the following research to achieve the baby monitoring, the temperature control and the humidity control in the infant incubator. The application of the IoT is rarely applied in the intelligent infant incubator for baby care and health monitoring. Compared with the traditional infant incubator, the intelligent infant incubator system proposed in this research provides the best environment and the most reliable care for the babies.

\section{THE OVERALL DESIGN FOR THE INFANT IN- CUBATOR INTELLIGENT CONTROL SYSTEM}

Considering the requirement for the parents and the doctors, the environment in the infant incubator should be monitored. And for the baby, the real-time situation of the body should be provided. The intelligent control system can selfadaptively change the environment by analyzing the feedback information by the sensors. What's more, when the parents want to have a look at their babies from work, it can be easily achieved by just opening their intelligent mobile phone. In the following, the overall design of the system is given.

\subsection{Overall Framework of the Intelligent System}

Considering the above requirement, the infant incubator intelligent system can be divided into three parts: the terminal device, the network and the baby monitoring and executive system. And the overall framework of the system is shown in Fig. (1).

As shown in Fig. (1), the first part mainly consists of the system computer or server, intelligent mobile phone and other terminal devices. And the second part is used to route and transmit the collected information and the control command, which includes the Internet and the local gateway.
The last part is the monitoring and execution system. In this part, the environment can be gotten by the sensors and the images and videos of the baby can be seen from the monitor. The relative information in the process is stored in the database. For example, if the baby's situation shows the bad trends through the judgment of the intelligent system, the execution system will begin to work to change the situation. At the same time, the danger signal is delivered to the host computer and the mobile phone of their parents by the alarm system and the system network.

\subsection{Introduction of the System Architecture}

Referring to the layer framework of the IoT, the proposed system is nearly divided into four layers. The layer framework of the intelligent system proposed in this application is presented in Fig. (2).

The layers will be explained as follows: The sensing layer is the lowest level of the whole structure. The temperature and humidity will be gotten by the corresponding sensors. And the real-time images and videos can be shown in the screen by the monitors or cameras. The sensors include the environmental sensors, GPS, cameras and the other sensing terminals. Furthermore, the controlling commands, such as turning up the air condition, can be controlled directly by the control module according to the real-time sensing values.

The network layer may include the ADSL, 3G or $4 \mathrm{G}$ mobile network and Zigbee and the intelligent local gateway will connect the network layer and the sensing layer. Specially, the technology of Zigbee is taken into account. By this device, the whole sensing network will be connected into the local gateway, and the Asymmetric Digital Subscriber Line (ADSL) is used to connect the system server to the local gateway, and the mobile network will be also used to pass the real-time information to the users or the host server. 


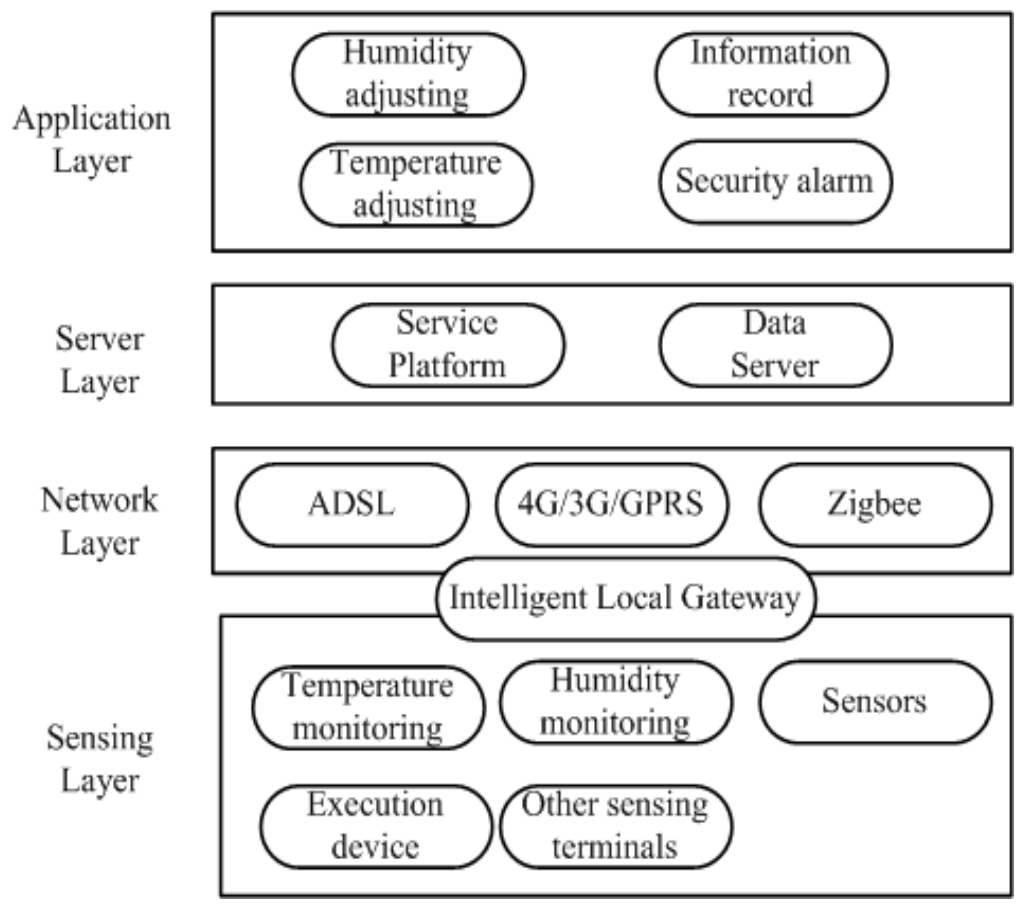

Fig. (2). The layer framework of the intelligent system.

As the responder of users' requests, the server layer consists of the service platform and the data server. In the service platform, Load balancing technology enhances the efficiency of system server. HTTP protocol is used to transmit data between the system server and both the intelligent local gateway client and the remote client. In data server, XML is used as data formulation. Database Input and Output (DBIO) API is designed to offer read and write interface for the system database.

The Application layer provides various services for both the local gateway client and the remote client with elegant system interface. Several function modules are designed in this layer, such as the humidity sensing module, temperature sensing module, security alarm and the process information record. All the data about the baby and the environment in infant incubator can be achieved by these modules to protect the baby from the bad condition and situation. This layer is the user interface of the infant incubator intelligent control system.

Based on the structure of the infant incubator intelligent system, the required functions can be obtained. The information about the baby can be achieved. In this way, the hardware design will be described in detail in the following research.

\section{DESIGN OF THE SYSTEM HARDWARE}

According to the required function of the infant incubator system, the hardware design is mainly divided into two parts. Firstly, it is the design of the intelligent local gateway. The other is the design of periphery nodes. The design mainly focuses on robustness and energy-saving of the intelligent infant incubator system.

\subsection{Design of the Intelligent Local Gateway}

The intelligent local gateway is deployed to construct and maintain the whole wireless automation network, as well as communicating with the system server. If some unsafe events happen in the infant incubator, such as the temperature is out of the allowed value and the baby is out of sight, warning messages will be sent to the system server and the mobile phone of the residents. Then, controlling command will be sent remotely to control electronic appliances and other smart components within the intelligent infant incubator. The design of the intelligent local gateway is illustrated in Fig. (3).

Android is installed for the intelligent control system which is an open source operation system that is widely used in the smart devices. Due to its character of open source, driver program for Zigbee technology can be simply installed on the intelligent system. With the Linux-based operation system for Zigbee nodes, various hardware drivers for the sensing and the controlling of these nodes can be easily installed. What's more, elegant and efficient system interface could be accomplished with Application Programming Interface (API) of Android. High compatibility of Android resulted in high appreciation all over the world. Finally, Android harbors an extensive application library, especially the seamless integration with Google Apps [9].

ARM11 is used as the hardware platform for the intelligent local gateway of the infant incubator system with the following advantages. Firstly, various modules, such as Zigbee communication module and 3G/4G/GPRS communication module, can be installed on ARM11 core board easily. Secondly, ARM11 launches $350 \sim 500 \mathrm{MHz}$ clock frequency 


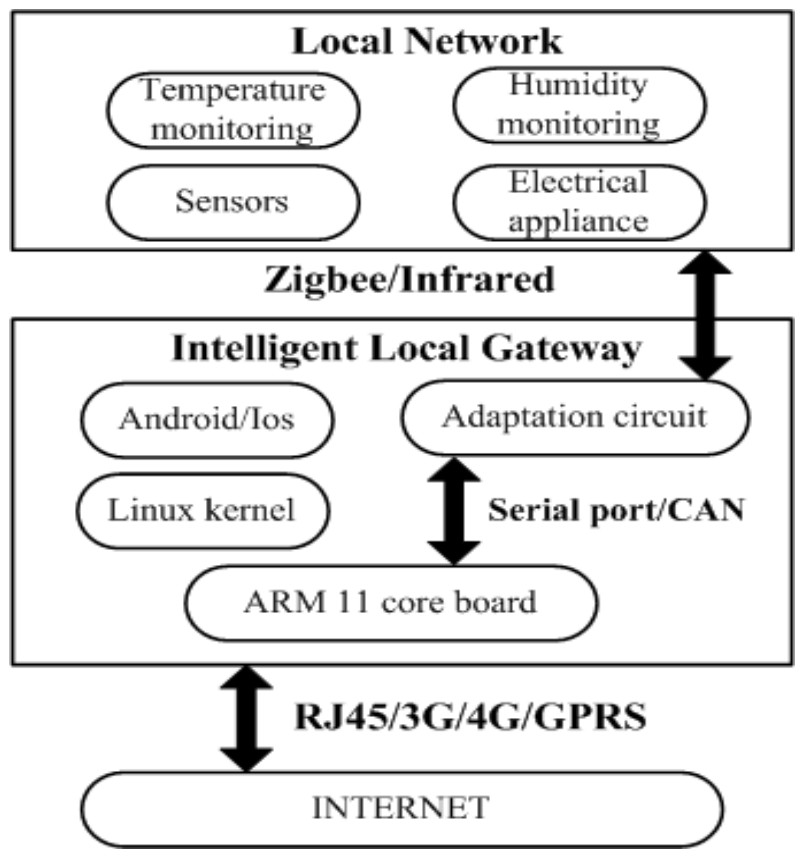

Fig. (3). The design of the intelligent local gateway.

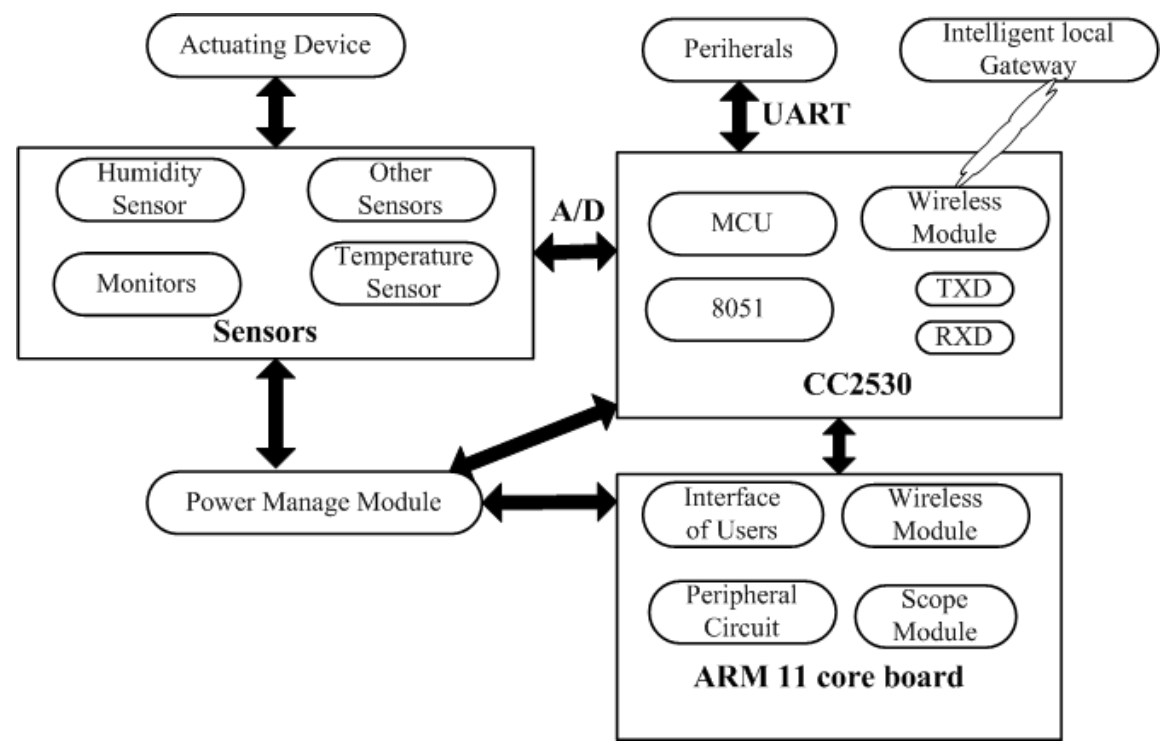

Fig. (4). The design of the periphery terminal.

kernel so that energy consumption could be reduced on the basis of adjusting the clock frequency and supply voltage dynamically. Finally, ARM11 processor offers good performance based on the multimedia processing instruction which can accelerate the processing of video and audio. And the new memory system of ARM11 improves performance of operating system as well. In the infant incubator system, a type of ARM1136J is taken into account. The corresponding data can be transmitted to the Internet by these methods.

The ZigBee module plays a role as a coordinator to maintain and manage the network. Then the CPU processes the coming signal and passes the signal to the USB modules by serial protocol.
The abilities of the local gateway are achieved by the extensible interface modules. For example a PCI card and RJ45 interface can be added on the module and connected to the Internet directly. The system can control the devices with infrared communication module by a wireless or repeater module.

\subsection{Design of the Periphery Terminal}

The hardware of detecting terminal mainly consists of the ZigBee module, the temperature sensors, the humidity sensor, relative execution devices and other peripherals. The architecture of detecting terminal is shown in Fig. (4). 
d (depth)

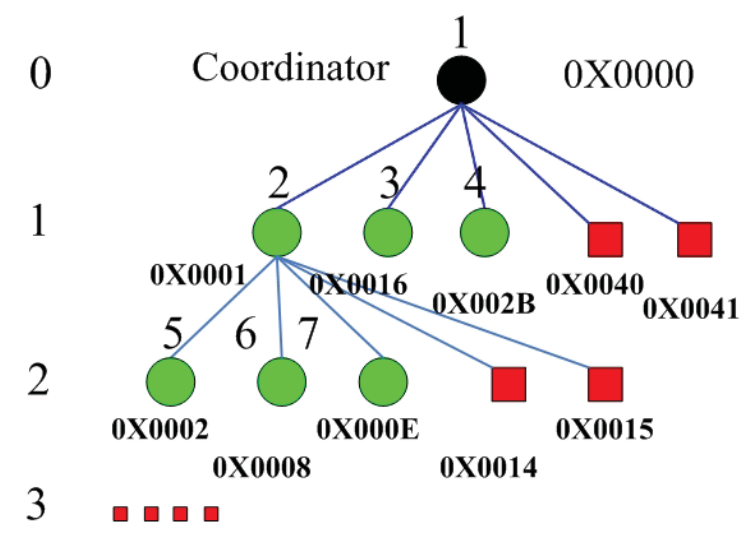

Fig. (5). An example of address allocation.

The periphery nodes are fixed for the sensing and the controlling of the intelligent infant incubator. Zigbee technology is used to construct and maintain the wireless home automation network. CC2530 is detected as hardware platform of Zigbee with its energy saving architecture.

The CC2530 chip is the kernel module of the system. The chip is specific to the IEEE 802.15.4 and Zigbee application of single chip solution, the economy and low power consumption. The CC2530 chip is made of a high performance $8051 \mathrm{MCU}, 2.4-\mathrm{GHz}$ RF transceiver. And what's more, it also includes the two powerful UARTs, 12-bit ADC with 8 channels and built-in ZigBee protocol stack. Power consumption can be reduced on the basis of four power modes and dynamic voltage adjustment. With the technical grade Zstack, the most competitive solution for Zigbee is provided by the CC2530 in the application areas.

According to the variable requirements for the perceptive information, infant incubator devices include different kinds of sensors, which are used for environmental monitoring and equipments controlling. The smart sensors are connected to MCU by the A/D module which can convert analog signals into digital signals.

Zigbee allocates each newly arrived node a unique network address by a distributed address allocation scheme. The coordinator, acted by the intelligent infant incubator gateway, of which is marked 0x0000 after initialization of network. And the other nodes will be allocated a network address consisting of three parameters, which are explained as follows.

$C_{m}$ is the maximum number of children of a parent node. And $R_{m}$ is the maximum number of routers of a parent node. $L_{m}$ is the maximum depth of the network.

$\operatorname{Cskip}(d)=\left\{\begin{array}{c}1+C_{m}\left(L_{m}-d-1\right) \quad \text { if } R_{m}=1 \\ \frac{1+C_{m}-R_{m}-C_{m} * R_{m}^{L_{m}-d-1}}{1-R_{m}}\end{array}\right.$

The address of the interval for neighboring nodes can be calculated as follows: And the parameter $d$ indicates router series, the $n$-order parent device address is acquired by Eq. (2).

$$
A_{\text {parent }}=0 \times 0000+(n-1) * \operatorname{Cskip}(d)
$$

The n-order wireless terminal address is obtained through Eq. (3).

$$
A_{n}=A_{\text {parent }}+\operatorname{Cskip}(d) * R_{m}+n
$$

Fig. (5) shows an example of address allocation with the parameters which are set as follows: $C_{m}=5, R_{m}=3, L_{m}=3$.

\section{THE SOFTWARE DESIGN}

According to the hardware design above, the software design includes the design of the sensing layer software and application support platform. The perception layer software is used for data collection and transmission of wireless sensor network. The application support platform promotes the development of intelligent infant incubator applications based on web and GUI (Graphical User Interface).

\subsection{The Design of the Perception Layer}

The software design of perception layer mainly includes the following four parts: the first is the initialization of the incubator hardware device, then building the network for the incubator system, thirdly, data procession and lastly event handing. According to the TI's Z-Stack, the software can be achieved by adopting the Embedded Workbench as a development tool in the application layer of Z-Stack [10]. The standard interface is written for the device to use. And the device definitions for different devices are also provided in the software. The process of the work is shown in Fig. (6).

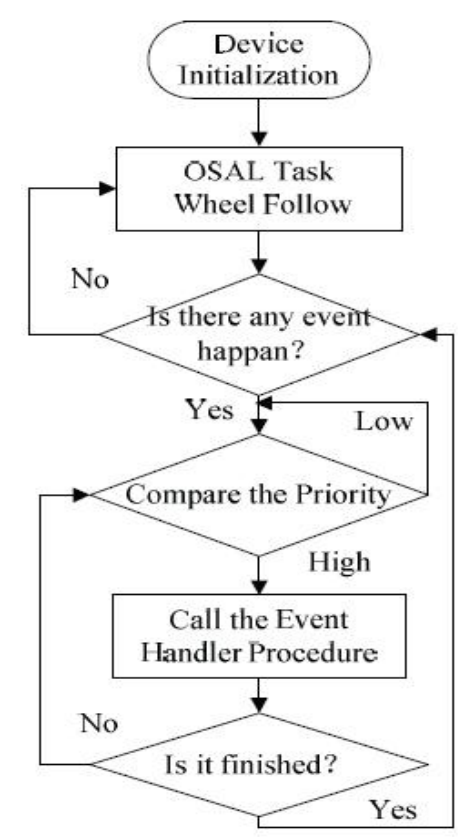

Fig. (6). The workflow of the software. 


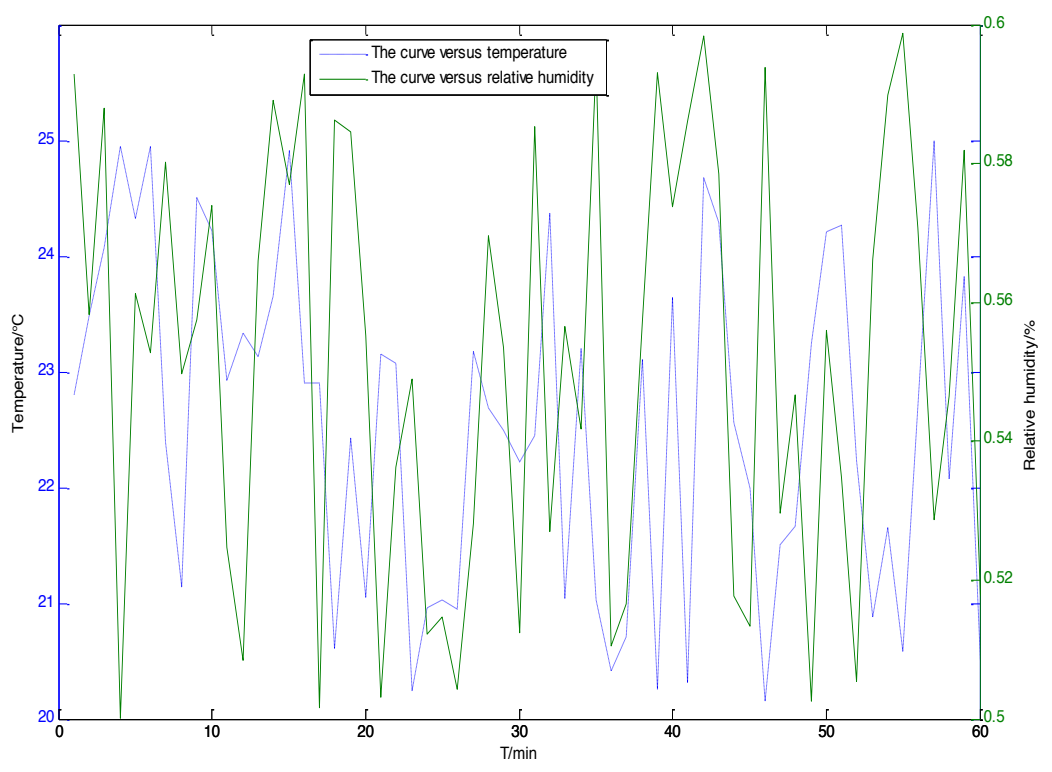

Fig. (7). The curve versus temperature and humidity in an hour.

The Initialization of devices; When a device connects to some network, it needs to be registered in the gateway and an address with 16 bit is assigned. The address is used to identify the equipments and the data in the network. According to the AODV (Ad hoc On demand Distance Vector) routing protocol for ad hoc networks, the Zigbee network is acquired. The agreement of promoting an environment can support mobile node, the connection fails and packet losses [11].

Task management in OSAL system: When the initialization of these devices above is completed, the function that can prepare and start the program is called to operate the OSAL system. It will monitor all task events and call the task event processor function for the task with the event. Function osalTaskAdd() aims at adding a task to the OSAL system.

Handing events; If there are events for a particular task, function will call the task event handler to handle events. Event was a deal with an event handler to the corresponding task. The events include timer events, operation events and response to events in the perception layer. Timer events update the environmental monitoring information by setting a timer on a regular basis. When the coordinator receives user control command, function will call a process routine operation event orders to handle events. Completion of the task response to the event handler is called after processing the received message.

\subsection{Software Design of Application Support Platform}

Software design of application support platform mainly includes web site creation and maintenance, the build and update of SQL database server and the command processing [12].

Web access; There are many web access methods that are available for choosing to achieve the web architecture the most famous methods are the $\mathrm{C} / \mathrm{S}$ mode and $\mathrm{B} / \mathrm{S}$ mode. However, with the $\mathrm{C} / \mathrm{S}$ mode environment faces many challenges. Therefore, $\mathrm{B} / \mathrm{S}$ architecture mode is selected to ensure that users can manage and control the systems at any time.

SQL database server; The database server is adopted to manage information of equipments, the users and the controlling methods. The Microsoft SQL Server which is a kind of database development tool is adopted to manage it. What's more, the ADO.Net data access technology exchanges data with database management system.

\subsection{The Experiment Results}

Through the design of the hardware and software for the intelligent infant incubator system, the information of the environment in the infant incubator can be obtained by the intelligent local gateway and the smart sensors.

Based on the above analysis, the curve of the temperature and humidity in a hour are shown in Fig. (7). We can easily find that the temperature is held in the range from $20{ }^{\circ} \mathrm{C}$ to $25^{\circ} \mathrm{C}$ under the control of the air-condition in the infant incubator. This range of the temperature is fit for the baby, and it is the same with the relative humidity in the infant incubator. The relative humidity is between $50 \%$ and $60 \%$. These data and experimental results show that the intelligent infant incubator system works well based on the design.

\section{CONCLUSION}

In this research, an intelligent infant incubator is proposed based on the IoT to achieve the remote monitoring and controlling of environment in the infant incubator such as temperature and humidity. The experimental results show that this system can provide a real-time and reliable management of the intelligent infant incubator system. Based on the analysis, a stable and robust security module should be 
included in the infant incubator intelligent control system. This will be the focus of the future work.

It is believed that with the development of IoT, the smart infant incubator will become more intelligent due to multiplicity.

\section{CONFLICT OF INTEREST}

The authors confirm that this article content has no conflict of interest.

\section{ACKNOWLEDGEMENTS}

Declared none.

\section{REFERENCES}

[1] A. Tellaeche, X.P. Burgos-Artizzu, G. Pajares, Á. Ribeiro, and C. Fernández Quintanilla, "A new vision based approach to differential spraying in precision agriculture", Comput. Electron.Agric., vol. 60, pp. 144-155, 2008.

[2] M. Zhang, B. Wang, C. Gao, and Q. Zhao, "Application study of precision agriculture based on ontology in the internet of things environment”, Commun. Comput. Inf. Sci., vol. 4, pp. 374-380, 2011.
[3] C. Zhang, and X.R. Miao, "Remote communication and control of smart home", Low Volt. App., vol. 3, pp. 20-22, 2012.

[4] H. Park, J. Burke, and M. B. Srivastava, "Intelligent lighting control using wireless sensor networks for media production", KSII Trans. Int. Inf. Syst., vol. 3, no. 5, pp. 423-443, 2009.

[5] Y.E. Duan, "Research on integrated information platform of agricultural supply chain management based on internet of things", $J$. Software, vol. 6, pp. 944-950, 2011.

[6] C.N. Verdouw, and A.J.M. Beulens, "Virtualisation of floricultural supply chains: A review from an internet of things perspective", Comput. Electron. Agric., vol. 99, pp. 160-175, 2013.

[7] H. Zhu, H. Yu, and F. Wang, "Design and implementation of sensor node for environment monitoring internet of things", Comp. Sci., vol. 39, pp. 126-129, 2012.

[8] X. Wang, "Application research of ecological environment monitoring based on internet of things technology", Transducer Microsyst. Technol., vol. 30, pp. 149-152, 2011.

[9] F. Zhang, "Research on applications of Internet of things in agriculture", Lecture Notes in Electrical Engineering, vol. 209, pp. 69-75, 2013.

[10] J.H. Wu, F. Ding, and Y.C. Chen, "Study on wireless data acquisition system of modern greenhouse", Comput. Meas. Control, vol. 15, no. 3, pp. 405-406, 2007.

[11] S.A. Brown, "Household technology adoption, use and impacts: Past, present and future", Inf. Syst. Front., vol. 10, no. 4, pp. 397 402, 2008.

[12] K. Cai, "Internet of things technology applied in field information monitoring", Adv. Inf. Sci. Ser. Sci., vol. 4, pp. 405-414, 2012.

(C) Huang and Sun; Licensee Bentham Open.

This is an open access article licensed under the terms of the Creative Commons Attribution Non-Commercial License (http://creativecommons.org/licenses/by$\mathrm{nc} / 4.0 /$ ) which permits unrestricted, non-commercial use, distribution and reproduction in any medium, provided the work is properly cited. 